\title{
Mixing methods and blending paradigms: some considerations for future research
}

\author{
Carolyn E. Schwartz $\cdot$ Dennis A. Revicki
}

Published online: 31 January 2012

(C) Springer Science+Business Media B.V. 2012

The past two decades have witnessed substantial advances in quantitative and qualitative methodological development. Supported by the growth in the capacity and application of computers, methodologists and computer scientists have developed software to make multivariate modeling and data mining more accessible and feasible. In the realm of qualitative analysis, a number of software programs now exist for analyzing content of coded qualitative data, allowing linguistic analysis [1] and thematic analysis [2] of large coded data sets. Such developments have set the stage for increasing sophistication in the melding of quantitative and qualitative methods to yield new insights into research questions across a range of disciplines, therapeutic areas, and aims.

This special section of Quality of Life Research was motivated by a desire to showcase the best and broadest applications of research that meld qualitative and quantitative methods. The process required setting clear criteria as standards, and ensuring that the peer review process adhered to those standards and was composed of reviewers with appropriate expertise in both qualitative and quantitative methods.

Over the course of developing this special section, two issues became clear. In the interest of furthering the field,

C. E. Schwartz $(\bowtie)$

DeltaQuest Foundation, Concord, MA, USA

e-mail: carolyn.schwartz@deltaquest.org

C. E. Schwartz

Departments of Medicine and Orthopaedic Surgery,

School of Medicine, Tufts University, Boston, MA, USA

D. A. Revicki

United Biosource Corporation, Bethesda, MD, USA we would like to describe these issues briefly. First, qualitative and quantitative methods derive not only from different complements of fields and foci, but in their origin were motivated by different types of research questions. In attempting to bring the two types of methods together, one faces a possible conflict of different paradigms. The qualitative paradigm seeks to describe a context or situation, with an aim to generate hypotheses and/or provide a beginning to an empirical foundation for new research questions. As part of instrument development, these qualitative methods are used to identify important and relevant concepts for measurement and for generating item content to cover these concepts. The concept of "generalizability" is often not relevant to the task at hand, as much as understanding what new concepts need to be further explored.

In contrast, quantitative research often focuses on sampling from a population and making inferences about the population based on the information about the sample. For example, research might seek to increase an understanding of patient responses that result in barriers to using specific treatments or differences in attitudes about research. In this context, generalizability is not only relevant but is a key aspect of validity (i.e., external validity).

The problem that we have seen often arise with qualitative research is that the sample size standards are generally relatively small, and thus, it is not possible to make statements about relationships between variables based on the sample. Nonetheless, such research may still use the language of inference and generalizability. Perhaps this language is an acquired habit from one's scientific training. Use of such language, however, muddies the water: are we generating hypotheses or testing hypotheses?

Our second reflection is related to the first issue. As one moves into the combined application of mixed methods, it 
would seem necessary to adopt a new paradigm and taxonomy. If one seeks "saturation" of a construct, for example, one would need to understand that the restrictedness of the sample-either in homogeneity or in sizewill necessarily reduce one's ability to make clear statements about saturation. If one interviews only Caucasian middle-class women, one may reach saturation of a concept, but it will only reflect this unique subgroup of people. If one seeks to reach saturation of the concept across a range of socioeconomic, ethnic, and experience backgrounds, then one will need to increase the number of strata, the size of the sample, etc. Fortunately, in some contexts, saturation is often reached with small numbers of participants (i.e., 7-8) (Willis [3]) so extending diversity may not require very large samples. In larger samples, quantitative methods, such as differential item functioning $[4,5]$, can be used to evaluate whether item responses differ by different socioeconomic or cultural groups.

This special section includes mixed methods applied to a range of research questions: instrument development, theoretical model development, utilities, methodological validation, clinical use of quality-of-life measures, and understanding treatment outcomes. It also includes a brief version of an NIH White Paper that was published recently on this methodological innovation. It is our hope that the special section provides models of the use of such methods for a range of research problems and paradigms in health outcomes research.

\section{References}

1. Pennebaker, J. W., Mehl, M. R., \& Niederhoffer, K. G. (2003). Psychological aspects of natural language use: Our words, our selves. Annual Review of Psychology, 54, 547-577.

2. QSR International. Available from: http://www.qsrinternational. com/about-qsr.aspx.

3. Willis, G. (2005). Cognitive interviewing. Thousand Oaks: Sage.

4. Teresi, J. A. (2006). Different approaches to differential item functioning in health applications advantages, disadvantages and some neglected topics. Medical Care, 44, S152-S170.

5. Teresi, J. A., \& Fleishman, J. A. (2007). Differential item functioning and health assessment. Quality of Life Research, 16(Suppl 1), 33-42. 